\title{
Philosophiques
}

\section{Lukasiewicz : de l'aristotélisme autrichien à l'aristotélisme polonais}

\section{Roger Pouivet}

Volume 26, numéro 2, automne 1999

La critique de la raison en Europe centrale

URI : https://id.erudit.org/iderudit/004970ar

DOI : https://doi.org/10.7202/004970ar

Aller au sommaire du numéro

\section{Éditeur(s)}

Société de philosophie du Québec

ISSN

0316-2923 (imprimé)

1492-1391 (numérique)

Découvrir la revue

Citer cet article

Pouivet, R. (1999). Lukasiewicz : de l'aristotélisme autrichien à l'aristotélisme polonais. Philosophiques, 26(2), 263-277. https://doi.org/10.7202/004970ar
Résumé de l'article

En 1910, Jan Lukasiewicz publiait Du principe de contradiction chez Aristote. Dans cet article, on explique les points principaux du livre de Lukasiewicz. Ce dernier affirme qu'Aristote n'a pas réussi dans sa tentative pour justifier le principe de contradiction. En fait, ce principe est moins logique qu'éthique, selon Lukasiewicz, et cela explique bien des difficultés posées par la théorie d'Aristote. On discute également de la façon dont Lukasiewicz utilise la notion d'« objets contradictoires », empruntée à la Théorie des Objets de Meinong; on montre que Lukasiewicz se situe dans le cadre d'une version brentanienne de l'aristotélisme. Certaines connexions entre Lukasiewicz et la conception wittgensteinienne de la nécessité ou le conservatisme logique de Quine sont indiquées. Le but de mon article est essentiellement d'encourager une lecture attentive du livre qui n’a pas reçu l'attention qu'il mérite parce qu'il a été écrit à l'origine en polonais. Souvent, les philosophes croient connaître son contenu à travers le résumé que Lukasiewicz écrivit en allemand en 1910, et qui a été traduit en anglais. Mais, en fait, il y a bien des choses importantes dans le livre qui n’apparaissent nullement dans le résumé. 


\title{
Lukasiewicz : de l'aristotélisme autrichien à l'aristotélisme polonais
}

\author{
ROGER POUIVET \\ Université de Rennes I \\ Roger.Pouivet@univ-rennesı.fr
}

\begin{abstract}
RÉSUMÉ. - En 1910, Jan Lukasiewicz publiait Du principe de contradiction chez Aristote. Dans cet article, on explique les points principaux du livre de Lukasiewicz. Ce dernier affirme qu'Aristote n'a pas réussi dans sa tentative pour justifier le principe de contradiction. En fait, ce principe est moins logique qu'éthique, selon Lukasiewicz, et cela explique bien des difficultés posées par la théorie d'Aristote. On discute également de la façon dont Lukasiewicz utilise la notion d'《objets contradictoires», empruntée à la Théorie des Objets de Meinong ; on montre que Lukasiewicz se situe dans le cadre d'une version brentanienne de l'aristotélisme. Certaines connexions entre Lukasiewicz et la conception wittgensteinienne de la nécessité ou le conservatisme logique de Quine sont indiquées. Le but de mon article est essentiellement d'encourager une lecture attentive du livre qui n'a pas reçu l'attention qu'il mérite parce qu'il a été écrit à l'origine en polonais. Souvent, les philosophes croient connaître son contenu à travers le résumé que Lukasiewicz écrivit en allemand en 1910, et qui a été traduit en anglais. Mais, en fait, il y a bien des choses importantes dans le livre qui n'apparaissent nullement dans le résumé.
\end{abstract}

\begin{abstract}
In 1910, Jan Lukasiewicz published On the Principle of Contradiction in Aristotle. In the present paper, I explain the main points in Lukasiewicz's book. Lukasiewicz claims that Aristotle was not successful in his attempt to justify the principle of contradiction. In fact, this principle is less logical than ethical, according to Lukasiewicz; and this accounts for many of Aristotle's difficulties. Lukasiewicz's use of the notion of "contradictory objects", taken from Meinong's Theory of Objects, is also discussed, and Lukasiewicz is shown to be situated within the Brentanian form of Aristotelism. I also indicate some connections between Lukasiewicz and Wittgenstein on necessity, and between Lukasiewicz and Quine on logical conservatism. The goal of the present paper is principally to encourage an attentive reading of a book that has not received the consideration it deserves because it was initially written in Polish. Often, philosophers believe that they grasp the content of the book through the brief abstract which Lukasiewicz wrote in German in 1910, and which has been translated into English ; but in fact there is much of importance which the abstract does not cover
\end{abstract}

\section{Aristotélisme autrichien et aristotélisme polonais}

L'interlocuteur privilégié des philosophes autrichiens du dix-neuvième siècle et du début du vingtième siècle a été A ristote. $0 \mathrm{n}$ peut ainsi parler $\mathrm{d}^{\prime}$ '« aristotélisme autrichien ». Dans cet essai, on commencera par définir succinctement trois attitudes générales à l'égard de la philoso phie d'A ristote, puisque l'une d'elles est justement caractéristique de l'aristotélisme autri- 
chien ${ }^{1}$. Puis on insistera sur l'importance du livre de Lukasiewicz D u principe de contradiction chez A ristote $(1910)^{2}$, un livre qui est, à mon sens, quelque peu sous-estimé. II est pourtant fondamental dans le passage d'un aristotélisme autrichien à un aristotélisme polonais.

Toute cette étude relève d'un intérêt plus large pour les figures de l'aristotélisme. ${ }^{3}$ L'aristotélisme polonais, de Twardowski à K otarbinski, en passant par Lukasiewicz, tout en se situant à coup sûr dans la lignée de l'aristotélisme autrichien, fait pendant à l'aristotélisme britannique tel qu'il se manifeste aujourd'hui chez G each, Anscombe, Kenny ou Wiggins. O n verra aussi que certaines remarques de W ittgenstein, dans lesquelles les aristotéliciens et thomistes britanniques ont reconnu les pensées du Stagirite et de l'A quinate, sont anticipées par Lukasiewicz dans son livre de 1910.

\section{Trois attitudes à l'égard d'Aristote}

La philosophie moderne est anti-aristotélicienne en ce qu'elle est post-cartésienne. Avec D escartes est apparue la thèse selon laquelle l'idée, comme représentation, s'interpose entre moi et le monde. Tout le problème métaphysique devient celui de savoir comment l'idée peut représenter correctement le monde et ce qui peut justifier ${ }^{4}$ la confiance que nous avons dans nos représentations. A ristote n'a pas grand-chose à dire sur ce point, puisque

1. Voir Smith, 1994, chap. 10, §6. Le réalisme autrichien peut être caractérisé de la façon suivante :

a) Le monde existe indépendamment de nous.

b) II existe des structures déterminantes réelles des choses (natures, lois), instanciées dans le monde.

c) N otre expérience des choses suppose à la fois un aspect singulier et un aspect universel ou général.

d) N otre connaissance est faillible et il n'y a pas de mode spécial et absolu de connaissance, même concernant l'aspect universel de notre connaissance.

e) Cependant, nous pouvons savoir, par le sens commun et par la connaissance scientifique, comment est le monde.

2. Toutes les références de Lukasiewicz sont des références à ce livre. Dans la mesure où il n'en existe pas de traduction française publiée, j'ai donné des références aux chapitres plutôt qu'aux pages de l'original polonais dont l'édition récente est due à Jan Wolenski.

3. Sujet auquel j'ai déjà consacré un ouvrage : Pouivet, 1997.

4. Le problème de la justification de notre connaissance est différent de celui de la garantie de notre connaissance. Le premier est typiquement issu du scepticisme moderne postcartésien. On se demande ce qui pourrait justifier une connaissance qui ne l'est pas. À défaut de cette justification, notre connaissance n'aurait qu'une valeur cognitive limitée. Le problème de la garantie part du fait de la connaissance, et cherche à découvrir pourquoi notre connaissance est garantie par le processus même par lequel elle se met en place. Justifier, c'est supposer que la connaissance est en question et le reste tant que la certitude n'a pas été constituée métaphysiquement, par une procédure du type de celle qu'on trouve dans les $M$ éditations métaphysiques. Garantir, c'est présenter la métaphysique de la connaissance comme une entreprise descriptive (et non pas d'abord normative) de l'activité cognitive et des objets d'une connaissance humaine garantie. 
pour lui le problème métaphysique consiste à s'interroger sur la nature de ce qui est, commel'indiquent les premières lignes du livre $\Gamma$ de la $M$ étaphysique. La métaphysique post-cartésienne est une machine de guerre contre un scepticisme qui n'est jamais pris au sérieux dans l'aristotélisme. La connaissance est un fait, et sa condition de possibilité n'a pas à être établie. La première attitude possible à l'égard d'A risto te dans la philoso phie moderne est ainsi le rejet d'une métaphysique qui ne prend pas au sérieux le défi sceptique de la correspondance entre nos représentations et la réalité. II est clair que l'aristotélisme qui s'est développé dans la philosophie autrichienne et chez Lukasiewicz échappe totalement à cette attitude. $\mathrm{N}$ on pas que l'argumentaire cartésien à propos de la représentativité des idées soit ignoré, mais on n'en tire pas de conséquence idéaliste.

L'autre attitude à l'égard de l'aristotélisme, c'est l'anti-modernisme. La scolastique cartésienne qui s'est développée aux $X V I^{e}$ et $X V I I I^{e}$ siècles ne peut pas vraiment contrecarrer les effets anti-religieux du scepticisme que la métaphysique idéaliste était supposée combattre. La métaphysique moderne, en tant qu'idéal isme et comme machine de guerre contre le scepticisme, si elle échoue, promeut cela même qu'elle combat. Inquiète d'un scepticisme qui conduit à l'agnosticisme ou à un christianisme personnel refusant l'autorité ecclésiale (et rejeton spiritualiste du kantisme ${ }^{5}$ ), la hiérarchie de l'Église catholique réagit en affirmant un thomisme strict. L'encyclique A eterni Patris de Léon XIII, le 4 août 1879, encourage le renouveau del'aristotélisme à travers le thomisme. Sans entrer dans le détail de la querelle moderniste, il apparaît hélas que le caractère réactif de l'aristotélisme néothomiste aura plutôt nui à l'aristotélisme que le contraire.

II y a cependant une troisième attitude possible à l'égard d'A ristote et de l'aristotélisme chrétien en général. C'est l'attitude des thomistes britanniques, mais c'est aussi celle de Brentano, dans ses travaux sur la métaphysique et la psychologie d'A ristote ${ }^{6}$. A ristote n'y est pas un simple repoussoir permettant de se démarquer d'une métaphysique réaliste et d'une philosophie de l'esprit non dualiste toutes deux parfaitement naïves, c'est l'interlocuteur philosophique de base. A ristote n'est pas une étape dépassée de l'H istoire de I'Esprit, c'est un contemporain.

Au tout début de sa carrière, Lukasiewicz écrivait dans un article consacré au concept de cause : « N i Kant ni H ume ne savait ce qu'est la métaphysique [...]. Les combats de $\mathrm{H}$ ume et de Kant contre la métaphysique n'étaient pas de véritables combats ; leurs traités n'indiquent pas qu'ils aient jamais examiné ce que disait A ristote. ${ }^{7}$ Ce passage peut être compris de deux façons. (a) L'attaque contre la métaphysique aristotélicienne par des philosophes représentatifs de la pensée moderne, comme $\mathrm{H}$ ume et $\mathrm{K}$ ant,

5. Voir Colin, 1997.

6. Voir pour une présentation Smith, 1994.

7. Cité par Skolimowski, 1967, p. 58. 
n'est pas victorieuse parce qu'elle ne porte pas contre ce qu'est vraiment cette métaphysique. (b) L'attaque contre la métaphysique aristotélicienne doit se faire sur d'autres bases que celles des philosophies de H ume et de Kant. II est vraisemblable que ce passage ait les deux significations. Lukasiewicz ne conçoit pas le respect philosophique pour les auteurs philosophiques autrement que sur le mode de la critique constructive, à l'instar de Brentano. Dès lors, prendre au sérieux A ristote, c'est juger de la réussite de ses projets philosophiques à l'aune des exigences d'A ristote lui-même. C'est aussi tenter d'améliorer le modèle légué, d'en résoudre les difficultés ou d'en montrer les impasses.

Cette attitude d'un aristotélisme constructif (plutôt que réactif) aura été celle de Brentano, de Lukasiewicz, mais aussi entre les deux guerres du Cercle de Cracovie. Des philosophes comme Salamucha ou Bochenski, prêtres catholiques, développent un aristotélisme qui use des instruments nouveaux de la logique moderne. ${ }^{8}$ Ils ne voient pas en celle-ci l'ennemi de la logique aristotélicienne, mais son renouveau, après les errements idéalistes, et tout particulièrement les prétentions de la dialectique hégélienne. Selon $B$. Smith,

Brentano a développé une conception de la science et de la connaissance qui incorpore à la fois des aspects de la philosophie cartésienne et de la philosophie empiriste britannique. Le contexte général de tous les écrits de Brentano est cependant la psychologie d'A ristote, avec l'ontologie dela substance matérielle et immatérielle qui va avec. ${ }^{9}$

L'aristotélisme polonais est plus radical encore. L'anti-psychologisme de Lukasiewicz est tel que les aspects du mentalisme cartésien qu'on trouvait encore chez Brentano disparaissent. Lukasiewicz ne fait aucun cas de l'idée cartésienne sel on laquelle la métaphysique consiste fondamentalement à examiner des contenus mentaux et à évaluer leur valeur fondationnelle pour la connaissance. C'est à l'évidence une idée totalement étrangère à l'aristotélisme. En revanche, cette idée, qui joue encore un rôle fondamental chez Brentano, sera développée par H usserl, malgré l'anti-psychologisme proclamé, en partie contre Brentano justement. Cette même idée se retrouve dans les différents courants phénoménologiques, avec une teneur variable en mentalisme et en idéalisme (très faible chez Ingarden, extrêmement forte

8. Pour Wolenski, 1989, « de nombreux philosophes de l'École de Lvov-Varsovie n'étaient pas seulement des catholiques romains, mais aussi bien des philosophes catholiques » (p. 16-17). Le Cercle de Cracovie comprenait aussi deux laïcs, D rewnowski, un ancien élève de Kotarbinski, et Sobicinski, qui était l'assistant de Lesniewski. Lors de leur première réunion (en 1936, Iors du Troisième Congrès de Philosophie à Cracovie), Lukasiewicz fit la première conférence (voir une version étendue de ce texte dans Lukasiewicz, 1970, «In Defense of Logistic »). Sur le Cercle de Cracovie, voir Bochenski, 1990, p. 19-21, et Puciato, 1993. Les travaux de ce $C$ ercle constituent un moment important, et trop méconnu, du thomisme au $X \mathrm{X}^{\mathrm{e}}$ siècle.

9. Smith, 1994, p. 35. 
chez certains phénoménologues français). Lukasiewicz renoue quant à lui avec un aristotélisme radical dans lequel l'analyse philosophique porte sur les objets de la connaissance et non pas sur les modes de la connaissance. II y a ainsi chez les Polonais, avec Lukasiewicz, mais aussi avec Kotarbinski, une descendance non phénoménologique de Brentano.

\section{Lukasiewicz est-il un crypto-idéaliste?}

En 1910, Lukasiewicz publie son premier livre, D u principe de contradiction chez A ristote. II en propose une sorte de résumé dans un article publié en allemand par le Bulletin international de l'A cadémie des sciences de Cracovie. II sera traduit en anglais en 1971 dans la R eview of M etaphysics (95). Du livre de 1910, il existe une traduction très récente en allemand. Une traduction encore plus récente en français est encore inédite. II reste que pendant très longtemps, ceux qui ne pouvaient le lire en polonais ne connaissaient le contenu du livre que par son résumé, à vrai dire assez brutal. D ès lors, on trouve deux types de considérations à l'égard de l'ouvrage. Dans le premier, l'ouvrage est cité comme anticipant les préoccupations futures de Lukasiewicz, aussi bien concernant la logique trivalente que la question des rapports entre déterminisme et liberté autour de la question des futurs contingents. L'ouvrage est plus mentionné et salué qu'examiné dans le détail. En revanche, dans le deuxième type de considérations, l'ouvrage est critiqué. $C$ 'est ainsi que Lukasiewicz est présenté, par les traducteurs français du résumé, comme faisant siennes les thèses des Sophistes ${ }^{10}$, et comme représentant le mode de pensée logico-scientiste auquel s'est opposé H eidegger ${ }^{11}$. Sel on cette interprétation, Lukasiewicz n'aurait à peu près rien compris à ce que cherchait à faire A ristote dans le livre $\Gamma$. II n'aurait pas saisi que « le principe de contradiction [est] inscrit d'avance dans la constitution du sens, [qu']il est comme le transcendantal de tout acte de parole, inscrit au cœur même de la langue ${ }^{12}$. Curieusement, Lukasiewicz est présenté comme défendant une doctrine supposée d'inspiration cartésienne selon laquelle le principe de la logique symbolique moderne consisterait dans un performatif par lequel le moi désignerait comme vraies certaines propositions.

Cette dernière thèse peut pourtant difficilement être attribuée à un philosophe archiréaliste, et dont l'anti-psychologisme a presque été obsessionnel. Ce que dit Lukasiewicz dans le passage incriminé du résumé (§ 10) ne

10. Cassin et $\mathrm{N}$ arcy, 1991, p. 9.

11. Cassin, 1989, p.12. Cette interprétation est particulièrement discutable puisque Lukasiewicz a toujours défendu le principe d'une indépendance de la logique et de la philosophie. Un système de logique est un instrument intellectuel sans rapport direct avec la réalité ; il peut être utile ou pas pour la réflexion métaphysique, mais en aucun cas il ne constitue un critère de correction philosophique. Cet aspect est très bien expliqué par F. Caujolle, 1970, p. 50-51.

12. Cassin et $\mathrm{N}$ arcy, 1991, p. 11. 
vaut que pour des jugements a priori et ne peut être interprété en termes d'une théorie générale de la vérité. II s'agit en fait d'une théorie de la définition. Si quelqu'un dit " J'entends par cercle une courbe », par le seul fait de le dire, il détermine cela même dont il parle ${ }^{13}$. M ême si Lukasiewicz nele précise pas, ce serait exactement la même chose si je disais que j'entends par licorne une bête ressemblant à un cheval, mais qui a une unique corne. Une telle définition peut s'avérer parfaitement inutile. C'est le cas, si rien ne correspond dans la réalité à ce dont je parle, et si nous ne sommes pas dans une fiction.

Par ailleurs, la conception de la vérité défendue par Lukasiewicz se situe dans la lignée de celle de Twardowski ${ }^{14}$, c'est-à-dire dans la lignée d'une théorie correspondantiste tout à fait aristotélicienne, comme peut s'en convaincre à la lecture du chapitre XVII du livre de Lukasiewicz. Dire alors, comme les traducteurs français du résumé, que cela « place curieusement tout l'édifice de la logique symbolique dans la dépendance du sujet - non du sujet transcendantal, mais du sujet actuel $»^{15}$, paraît vraiment discutable.

Toute cette interprétation négative des thèses de Lukasiewicz est partiellement due au fait de juger de ce que dit un philosophe dans un livre très dense de 120 pages par l'esquisse d'une vingtaine de pages qu'il en a proposée à seule fin de faire lire le livre. L'esquisse consiste même très largement en citations en grec d'A ristote et dans leurs traductions allemandes. Cette interprétation souffre également du manque de considération pour l'ancrage autrichien et polonais de Lukasiewicz dont il a été question dans le $\S 2$. Enfin, cette interprétation sous-estime beaucoup l'importance du fait que lorsque Lukasiewicz rédige le livre de 1910, il conçoit encore l'apport de la logiqueà l'analyse philosophique comme une garantie de rigueur et de clarté, tout comme Bolzano ou Brentano. II n'a pas encore accordé au calcul propositionnel l'importance qu'il lui donnera à partir de 1913, et qui le conduira alors, et alors seulement, à faire de la logique un instrument de critique des faux problèmes philosophiques, une attitude philosophique qu'on retrouvera aussi chez le Wittgenstein du Tractatus. ${ }^{16}$ En 1910, Lukasiewicz fait de la philosophie "scientifique», comme disent les Polonais, ou " analytique », comme disent les Anglo-Saxons. C'est seulement à partir de 1926, alors qu'il n'enseigne plus que la logique mathématique, que s'accentue ce mode de pensée pour lequel la logique est effectivement la pierre de touche ultime. En 1910, Lukasiewicz ne dit rien de plus que ce que disent A ristote ou le philosophe analytique typique d'aujourd'hui : la logique constitue

13. Lukasiewicz, 1910a, chap. VIII

14. Voir Smith, 1994, chap. 6, § 2.

15. Cassin et $\mathrm{N}$ arcy, 1991, p. 11

16. À cet égard, les affinités de Lukasiewicz avec les membres du Cercle de Cracovie, des théistes convaincus et militants, invalident complètement l'idée d'un Lukasiewicz positiviste, au moins dans une acception courante du terme. D ans le Cercle de C racovie, la logique est mise au service de la métaphysique et de la théologie, et ne joue nullement contre elles. 
I'organon de la philosophie, ce grâce à quoi on peut espérer parvenir à des résultats fiables. Comment peut-on reprocher à Lukasiewicz de n'avoir pas compris A ristote alors même qu'il est, en 1910, d'un aristotélisme de stricte obédience, même s'il n'est pas confit de dévotion à l'égard du Stagirite? Surtout, comment peut-on le lui reprocher au nom d'un (supposé) principe transcendantal inscrit dans la langue et qu'A ristote aurait mis en évidence? C ela signifierait qu'A ristote ne serait pas réaliste, qu'il soutiendrait une sorte d'idéalisme linguistique - ce qui paraît tout de même assez peu probable...

\section{Le principe de contradiction comme principe éthique}

Pour Lukasiewicz, l'histoire du principe de contradiction connaît trois moments. D'abord, le combat d'A ristote contre les Sophistes, puis la contestation hégélienne, et enfin l'examen actuel, en 1910, de la possibilité d'une « logique non aristotélicienne ${ }^{17}, c^{\prime}$ est-à-dire omettant le principe de contradiction. Si le troisième moment est nécessaire, c'est que le deuxième était un faux-semblant. H egel réagissait à la thèse kantienne selon laquelle la métaphysique verse inévitablement dans des antinomies et s'enferre ainsi dans des contradictions ${ }^{18}$. H egel « reconnut l'existence réelle des contradictions en y voyant un élément de vie et mouvement ${ }^{19}$. M ais chez $\mathrm{H}$ egel, la contestation du principe de contradiction est « purement verbale » 20 ; elle ne s'appuie sur aucun travail sérieux d'examen du principe lui-même. Ainsi, il faut reprendre le travail entrepris par Aristote dans la M étaphysique. Le résultat sera l'établissement du principe de contradiction, alors même qu'A ristote pensait cela impossible ${ }^{21}$. Dès lors, l'établissement du principe indiquera sa vraie nature.

Lukasiewicz dit alors ce que W ittgenstein dira plus tard à propos de la nécessité : le principe de contradiction est commeun roc au-delà duquel nous ne pouvons plus creuser. Certes, on peut toujours construire des systèmes formels qui l'omettent. Lukasiewicz ajoute même que D ieu pourrait se passer de ce principe ${ }^{22}$. $M$ ais nous, humains, ne pouvons pas nous en passer. $N$ ous sommes ainsi faits. L'homme est non seulement faillible, mais menteur. Dès lors, pour décel er l'erreur et le mensonge, nous devons accepter le principe de contradiction. Seule la contradiction permet en effet de les révéler. $M$ ais s'il n'existait ni erreur ni mensonge à déceler, rencontrer une contradiction ne devrait conduire qu'à l'accepter sans mal. Autrement dit, il n'y a pas de raison logique ni ontologique à l'acceptation du principe de contradiction, mais une raison qui concernela nature humaine elle-même. $0 \mathrm{n}$ aurait donc tort de

\footnotetext{
17. Lukasiewicz, 1910a, chap. XVI.

18. Ibid., introduction.

19. Ibid., introduction.

20. Ibid., introduction.

21. M ét., Г, 6, 1011a 12.

22. Lukasiewicz, 1910a, chap. XX.
} 
penser que, dans le texte de 1910, Lukasiewicz conteste la validité du principe de contradiction. $\mathrm{O}$ n aurait également tort de penser que la validité du principe de contradiction, parce qu'elle est « pratique et éthique $»^{23} \mathrm{n}^{\prime}$ aurait dès lors aucune objectivité, qu'elle « détruit la nécessité éternelle et objective ${ }^{24}$ dont A ristote aurait paré le principe. Q ue la nécessité du principe de contradiction ne soit ni logique (il n'y en a pas de preuve logique), ni ontologique (la notion d'objets contradictoires n'est pas absurde), ne change rien à cette nécessité. $Q$ ue ce principe soit lié à une caractéristique de la nature humaine, la faillibilité, nele rend pas moins sérieux. M êmesi Lukasiewicz ne s'exprime nullement de cette façon, on pourrait considérer qu'il y a une nécessité anthropologique qui a trait à notre nature propre, et dont le principe de contradiction relève.

Lukasiewicz propose une expérience de pensée tout à fait similaire à celles auxquelles le second W ittgenstein nous a accoutumés ${ }^{25}$, celle d'une vie sociale sans le principe de contradiction.

Quelqu'un est injustement accusé du meurtre d'un ami. De faux témoins, déposant sous serment, déclarent avoir vu l'accusé le jour du crime au domicile de sa victime, avoir suivi de loin le déroulement de la dispute pour, enfin, se voir obligés d'assister à la triste scène finale sans pouvoir venir à temps au secours de la victime. L'accusé proteste de son innocence, s'en rapporte à sa vie irréprochable, à son caractère calme et conciliant, à l'amitié de longues années qui le liait avec le défunt; enfin il cite toute une liste de témoins dignes de confiance qui, d'une façon unanime et irréfutable, établissent son alibi. $M$ ais à quoi tout cela lui servira-t-il? II ne peut que justifier son propre propos selon lequel il n'a pas tué l'ami. Cependant, si le principe de contradiction n'existe pas, la vérité de ce jugement n'exclut pas la vérité du jugement contradictoire selon lequel il l'a tué. Aussi, ne disposant d'aucun moyen de réfuter un faux témoignage de gens indignes, le juge doit-il admettre que sans tuer son ami, l'accusé l'a quand même tué, à la suite de quoi une condamnation est prononcée. ${ }^{26}$

La contradiction, tant qu'on en reste dans les sciences formelles, la logique ou les mathématiques, n'est en rien un mal. Un paradoxe logique, comme celui de Russell par exemple, doit être reconnu et accepté. Reconnaître l'existence d'une contradiction est alors un progrès scientifique. Ce sont les sciences empiriques et la vie quotidiennequi ne peuvent tolérer l'omission du principe de contradiction. Pour Lukasiewicz, c'est la raison pour laquelle, en l'absence même de toute preuve au sens strict, A ristote élèvele principe de

23. Ibid., chap. $X X$.

24. Cassin et $\mathrm{N}$ arcy, 1991, p. 9.

25. Il dit aussi : «La fiction est le moyen scientifique le plus approprié pour illustrer l'importance des lois, des causes ou des propriétés des objets analysés, puisqu'elle permet, par exemple, d'abolir certaines lois gouvernant tel ou tel ensemble de phénomènes » (Lukasiewicz, 1910a, chap. XVI).

26. Lukasiewicz, 1910a, chap. XX. 
contradiction au rang de dogme. Les Sophistes ridiculisaient la science dans I'opinion publique et jetaient la confusion dans les esprits. Le principe de contradiction n'est en rien un principe dont la valeur serait logique ou ontologique, et encoremoins un transcendantal du sens, c'est un principeéthique.

\section{Ontologie, logique et psychologie}

Ce qui est le mieux connu dans Du principe de contradiction chez A ristote, c'est la distinction entre trois principes de contradiction à partir du texte de la M étaphysique ${ }^{27}$ :

- principe ontologique: Aucun objet ne peut en même temps posséder et ne pas posséder une même propriété ;

- principe logique : D eux jugements dont l'un attribue à l'objet justement cette propriété que l'autre lui refuse, ne peuvent être vrais en même temps ;

- principe psychologique: D eux convictions auxquelles correspondent des jugements contradictoires ne peuvent pas exister ensemble dans le même esprit.

$S^{\prime} i l$ s'agit bien de trois principes différents, c'est qu'ils portent sur des objets différents : les objets du monde, les jugements, les convictions. Ils ne sont pas synonymes, mais équivalents. Les deux premiers le sont du fait $d^{\prime}$ une correspondance entre l'être et les jugements vrais ${ }^{28}$. Sel on Lukasiewicz, A ristote ne tente pas de prouver les principes ontologique et logique ; il essaie simplement de prouver le principe psychologique en partant des deux autres sur le mode suivant :

Si deux convictions auxquelles correspondent des jugements contradictoires existaient dans le même esprit, cet esprit posséderait ensemble des propriétés contraires. Selon le principe logique de contradiction, aucun objet ne peut posséder ensemble de propriétés contraires. Dès lors, deux convictions auxquelles correspondent des jugements contradictoires ne peuvent pas exister ensemble dans le même esprit. ${ }^{29}$

Cela suppose que les convictions soient des propriétés de l'esprit dans lequel elles existent. Cela suppose aussi une relation de contrariété entre la conviction la plus vraie et la conviction la plus fausse. Pour Lukasiewicz, on aura alors des différences graduelles entre la vérité et la fausseté. $M$ ais pour qu'il y ait des degrés de vérité, il faudrait qu'il y ait des degrés d'inhérence des propriétés aux objets qui les possèdent. À défaut de cela, comment y auraitil des convictions contraires situées de chaque côté d'une relation de contrariété? C ette difficulté est due à la confusion entre logique et psychologie, c'est-à-dire à la thèse selon laquelle jugements et convictions entretiennent

27. Ibid., chap. I.

28. M ét., $\Gamma, 7,1011 b$ 26-27.

29. Lukasiewicz, 1910a, chap. III, i.f. 
les mêmes relations. En général, on pense en ce cas au psychologisme. $M$ ais ici Lukasiewicz en a plutôt contre le logicisme en psychologie, c'est-à-dire la thèse selon laquelle ce qui vaut pour les jugements, objectifs, vaut pour les convictions, subjectives. ( $D$ 'après Lukasiewicz, M einong n'est pas sans faire la même erreur.) Finalement, le principe psychologique de contradiction n'est nullement prouvé, parce qu'il dépend de l'expérience. En sa faveur, il n'est pas possible d'aller plus loin qu'une supposition inductive.

Lukasiewicz montre ensuite ${ }^{30}$ qu'on aurait tort de considérer le principe d'identité co mme une formulation positive du principe de contradiction. D'une façon générale, les trois principes d'identité, de contradiction et de double négation ne sont pas synonymes, même s'ils peuvent être considérés comme logiquement équivalents. Par exemple, si le principe de contradiction peut prendre systématiquement la forme d'un énoncé conditionnel concernant un objet $O$ ( « Si $O$ est un objet, alors $O$ ne peut pas posséder $P$ et ne pas posséder $P »)$, l'introduction de la notion d'objet dans la formulation du principe d'identiténe permet d'obtenir qu'un cas particulier du principe (« Si 0 est un objet, alors 0 est un objet/O ne peut pas ne pas être un objet »). $P$ est n'importequelle propriété d'un objet quelconque, alors que dans lecas de l'identité on a restreint le principe à la notion d'objet.

Lukasiewicz examine aussi la preuve élenctique et la preuve par réfutation. La M étaphysique n'est pas pour Lukasiewicz l'objet d'un commentaire, mais d'une discussion critique et d'une évaluation de l'argumentation qui ne s'embarrasse pas d'une interprétation historique. La question est de savoir si A ristote réussit à faire ce qu'il dit qu'il fait, c'est-à-dire à prouver le principe de contradiction. La réponse de Lukasiewicz est non. Autrement dit, il traite A ristote comme A ristote traitait ses prédécesseurs et ses contemporains. En cela, Lukasiewicz anticipe le rapport aux textes philosophiques canoniques qu'on trouve dans le livre de Russell sur Leibniz et qu'on retrouve dans la façon dont Strawson ou Bennett ont lu Kant ou Spinoza, ou celle dont Kenny a lu Descartes et saint Thomas.

Prenons ainsi l'exemple du chapitre $X$. Lukasiewicz discute le texte de M étaphysique, $\Gamma, 4,1006 \mathrm{~b}$ 28-34. Q uand on prononce le mot « homme», on entend par ce terme ceci ou cela. A ristote précise : " Si le mot homme et le mot non homme ne signifient pas des choses différentes, il est clair que n'être pas un homme a la même signification qu'être un homme, et que réciproquement être homme se confond avec n'être pas homme. »

Le raisonnement d'A ristote serait le suivant :

1) J'entends par $O$ quelque chose qui est $P$.

2) $O$ doit donc être $P$.

3) $O$ ne peut pas ne pas être $P$.

4) $O$ ne peut pas à la fois être $P$ et ne pas être $P$.

30. Ibid., chap. VII. 
La prémisse (3) est en fait le principe de double négation. Si selon (2) $O$ doit être $P$, alors $O$ ne peut pas ne pas être $P$. Autrement dit :

$S^{\prime}$ il est nécessaire que $O$ soit $P$, alors il est nécessaire que $O$ ne soit pas non $\mathrm{P}$.

Or, le principe de double négation n'implique pas le principe de contradiction, contrairement à ce que suggère A ristote. Les objets contradictoires, comme cercle carré, ne peuvent être considérés comme de simples associations de sons sans signification, comme abracadabra. Jusqu'à $\mathrm{H}$ ermite et Lindemann, au XIX ${ }^{\mathrm{e}}$ siècle, on a essayé de construire le carré dont la surface égalerait celle d'un cercle de rayon 1 . O n le sait maintenant, ce carré est un objet contradictoire, puisqu'il doit avoir des côtés qu'il est impossible d'exprimer par un nombre algébrique. II n'en est pas moins un objet, dit Lukasiewicz. Le principe de double négation s'applique à ce carré. Si c'est un carré, il doit avoir des côtés exprimables par un nombre algébrique, et donc il ne peut pas ne pas avoir des côtés exprimables par un nombre algébrique. Le principe de double négation s'applique, mais le carré en question, en tant qu'objet, a et n'a pas la propriété que ses côtés soient exprimables par un nombre algébrique. Autrement dit, le principe de double négation n'implique pas le principe de contradiction. Dès lors (1)-(3) n'implique pas (4). Lukasiewicz précise qu'on pourrait contester la notion même d'objet contradictoire, c'est-à-dire l'idée qu'il s'agisse d'objet à proprement parler. $M$ ais alors il faudrait une affirmation plus forte que celle qu'entend faire A ristote dans le passage incriminé. II faudrait une affirmation ontologique, et pas seulement une inférence qui prétend acculer le contradicteur à accepter cela même qu'il prétend rejeter, selon le principe d'une preuve élenctique.

On a là un exemple de la démarche de Lukasiewicz. Ce qui fait tout l'intérêt du livre, ce sont ces discussions détaillées, justement parce qu'elles prennent au sérieux les arguments d'A ristote en les discutant.

\section{Ontologie de la substance, objets constructionnels et reconstructionnels}

Le livre de 1910 peut être considéré comme anticipant largement ce qu'on peut appeler la métaphysique analytique, c'est-à-dire l'usage d'une instrumentation logique pour analyser les thèses métaphysiques - ce à quoi la lecture de Strawson, Q uine, Chisholm ou Armstrong nous a accoutumés.

Ainsi, dans le chapitre XI, L ukasiewicz insiste sur le lien étroit entre le principe de contradiction et I'ontologie aristotélicienne de la substance. Pour A ristote, les mots n'ont de sens que s'ils désignent quelque chose qui, dans son essence, est singulier ${ }^{31}$. Le principe de contradiction reposerait ainsi finalement sur l'impossibilité pour une chose d'être ce qu'elle est et à la fois de ne pas l'être. La conséquence serait que ce principe ne vaudrait finalement

31. M ét., $\Gamma, 1006 a$ 31-34; 1006b 7-9. 
pas pour les propriétés accidentelles. En cela, le principe de contradiction est un élément dans sa polémique contre les $M$ égariques, qui ne reconnaissent pas la différence entre substance et accident, mais égal ement contre Protagoras. Pour réfuter la thèse sel on laquelle tout est à la fois vrai et faux, A ristote propose la distinction entre actualité et potentialité : "Si une même chose peut tout à la fois être et n'être pas, ce n'est pas du moins dans le même sens. En puissance, une même chose peut être les deux contraires, mais en absolue réalité, elle nele peut pas. ${ }^{32}$ » Sel on Lukasiewicz, le principe de contradiction ne concernerait alors que les êtres actuels et non les êtres en tant que potentiel $s^{33}$. A ristote semble même dire que les objets de la perception sont des êtres potentiels ${ }^{34}$. Dans la mesure où ils disent qu'il n'y a que des objets de perception, les Sensualistes ont raison de ne pas admettre le principe de contradiction. Simplement, ils ont tort de croire qu'il n'existe que des objets de perception, et pas des substances. L'univocité des termes suppose des concepts des choses ; les concepts des choses supposent une nature propre des choses qui sont dans l'extension des concepts que les termes expriment. Lukasiewicz en conclut que « force est de constater que le principe de contradiction chez A ristote est non seulement un principe ontologique, mais qu'il revêt également un sens métaphysique ${ }^{35}$. Tout le problème est que reste obscur le lien exact entre la théorie aristotélicienne de la prédication singulière vraie, qui est au cœur de sa métaphysique, et le principe de contradiction. ${ }^{36}$

Lukasiewicz montre égal ement que « le principe du syllogisme et le raisonnement syllogistique seraient valables, même si le principe de contradiction était erroné ${ }^{37}$. Par exemple, le syllogisme suivant est parfaitement valide :

B est $A$ (et n'est pas en même temps non A)

C est et $n$ 'est pas $B$

$C$ est $A$ (et n'est pas en même temps non $A$ )

Lukasiewicz signale qu'A ristote savait cela ${ }^{38}$. II aurait donc pu en conclure qu'une communauté dans laquelle les négations seraient systématiquement vraies pourrait ainsi noter des faits d'expérience, raisonner inductivement et déductivement, et agir efficacement à partir de tels raisonnements. Il existe un monde possible, celui d'une logique non aristotéli-

32. M ét., $\Gamma, 5,1008 \mathrm{~b}$.

33. Lukasiewicz, 1910a, chap. XIV.

34. En 1010a 1-5.

35. Lukasiewicz, 1910a, chap. XIV.

36. Voir Anscombe, 1961, p. 41-46, pour une analyse serrée de ce point.

37. Lukasiewicz, 1910a, chap. XV.

38. Seconds A nalytiques, I, 11, 77a 10-22. 
cienne - le M onde des N on-A si l'on préfère - dans lequel le principe de contradiction ne vaut pas. Pour autant que les êtres qui y vivent ne fassent pas d'erreur et ne mentent pas, ils y vivent fort bien. $M$ ais certes, c'est un monde d'anges plus que d'êtres humains.

Pour pouvoir tirer le principe de contradiction d'une ontologie de la substance, encore faut-il limiter la notion d'objet aux objets non contradictoires. Lukasiewicz est ainsi conduit dans le chapitre X VIII de son livre à développer une ontologie des objets contradictoires. II part de la distinction meinongienne - il a suivi les cours de M einong à Graz en 1909 - entre objets complets, qui sont les objets concrets, et objets incomplets, qui sont les objets abstraits. Parmi les objets incomplets, il y a des objets reconstructionnels, c'est-à-dire des objets incomplets mais qui deviennent concrets par un complément adéquat. Ce sont les objets des notions empiriques, comme homme, plante, cristal, rayon, etc. II y a aussi des objets constructionnels. Ils ne peuvent être complétés et transformés en objets concrets. Ils ne dépendent pas de l'expérience. $\mathrm{O}$ les trouve dans les domaines de la logique et des mathématiques. Ces objets ne peuvent pas moins en jouer un rôle théorique et pratique. Dans la mesure où nous tenons au principe de contradiction, nous les construisons de façon qu'ils ne soient pas contradictoires. Cela n'empêche pas que de telles contradictions apparaissent, malgré nous. C'est le cas pour «le plus grand nombre premier ». Nous n'avons finalement aucune garantie qu'il existe des objets constructionnels non contradictoires. La construction des objets constructionnels est libre, mais les relations qu'ils entretiennent une fois construits sont indépendantes de nous, et peuvent provoquer des contradictions. L'exemple le plus fameux est celui du rapport entre la suite infinie des nombres entiers et la suite infinie des nombres pairs. La partie, la suite des nombres pairs, est égale au tout, la suite des nombres entiers. Lukasiewicz cite aussi le transfini et le paradoxe russellien de la classe de toutes les classes qui ne sont pas membres d'elles-mêmes.

Peut-il y avoir des objets reconstructionnels contradictoires? Lukasiewicz reconnaît aisément que nous sommes tentés de penser que non. Pourtant, il propose encore cette expérience de pensée :

Imaginons une coupe en travers du monde entier des phénomènes à un moment du temps. II n'y aurait plus aucun changement, le temps n'existerait plus à la surface figée de cette coupe, et la flèche [du temps] se trouverait figée en un endroit. $M$ ais pouvons-nous savoir si, effectivement, elle se situerait seulement en un endroit? $O r$, tant qu'elle se déplaçait, elle changeait constamment sa position dans l'espace et à chaque moment du temps continu, elle se trouvait dans plusieurs endroits. Pourquoi donc ne pourrait-elle pas être dans au moins deux endroits différents à un moment discontinu, c'est-à-dire à la fois se trouver dans un endroit et ne pas s'y trouver? Comment savoir si une contradiction semblable ne serait pas contenue dans chaque objet subissant un changement quelconque? Et puisque tout change constamment et tout s'écoule, il se pourrait que le monde sensoriel contienne de nombreuses contradictions révélées par une telle coupe. 
La contradiction, si elle concerne un moment discontinu auquel nous n'avons pas accès, est à la fois réelle et indécelable pour des êtres humains. II pourrait y avoir des objets concrets contradictoires, mêmes'il est impossible de prouver que de tels objets existent bien, et même si rien dans notre expérience ne conduit indubitablement à le penser.

\section{Révision minimale}

Lukasiewicz a soumis le livre $\Gamma$ de la M étaphysique d'A ristote à une critique radicale. La conclusion en est qu'il est fort possible que le principe de contradiction ne puisse au sens strict être prouvé. En cela, L ukasiewicz s'accorde avec une conception qu'on trouvera chez W ittgenstein : convention et nécessité ne sont pas du tout contradictoires. II se pourrait que le principe de contradiction ne puisse être prouvé, sans pour autant qu'il ne nous soit pas nécessaire de le respecter. II anticipe aussi la thèse quinéenne selon laquelle certains principes fondamentaux de la logique pourraient être contestés, mêmesi nous ferons toujours tout pour éviter leur rejet. Le coût cognitif d'un tel rejet serait beaucoup plus grand que tout effort de conservation du principe contestée ${ }^{39}:$ « $M$ ême si nous nous retrouvions un jour face à une contradiction réelle, bien évidemment, sans le savoir, il y aurait toujours des moyens pour l'éliminer. ${ }^{40} \mathrm{O}$ n interpréterait l'expérience de façon telle qu'une révision minimale, excluant surtout le principe de contradiction, résoudrait le problème. Si nous trouvons un objet concret contradictoire, nous pouvons toujours dire qu'il s'agit en fait de deux objets.

Lukasiewicz constate ainsi que ce qui nous est nécessaire pourrait bien cependant n'être pas une loi de l'être. N ous ne pouvons connaître a priori les lois divines, précise-t-il ${ }^{41}$. Le principe de contradiction, plus qu'une loi absolue et indiscutable s'imposant à toutes choses, ne constitue qu'une contrepartie épistémique d'une nature humaine faillible. II paraît alors clair que la critique à laquelle A ristote est soumis relève, typiquement, de l'aristotélisme chrétien. Ce que Lukasiewicz dit, c'est que la nature humaine est aussi celle d'un être limité, d'une créature.

Cen'est pas parce que nous ne pouvons pas, nous, créatures, nous passer du principe de contradiction qu'il faut lui accorder le statut d'un principe absolu, le statut d'une vérité éternelle s'imposant à Dieu lui-même.

Bydgoszcz, août 1997 - Saint-Laurent, janvier 1998

39. Par exemple, Q uine et Ullian, 1970, pour une présentation simple de cette thèse majeure de Q uine. Voir aussi sur cette question, quand il s'agit de logique, H aack, 1996.

40. Lukasiewicz, 1910a, chap. XIX.

41. Ibid., chap. XX. 


\section{Bibliographie}

Anscombe, G.E.M . et Geach, P., Three philosophers: Aristotle, Aquinas, Frege, Londres, Blackwell, 1961

Bochenski, J.-M., Entre la foi et la logique, Entretiens avec Joseph-M. Bochenski recueillis par J. Parys (traduction française : M orin-A guilar, E., Paris, Les éditions N oir sur Blanc, 1990)

Cassin, B., « Parle si tu es un homme », introduction d'une traduction et d'un commentaire du livre $\Gamma$ de la $M$ étaphysique, sous le titre $L$ a décision du sens, Paris, Vrin, 1989

Cassin, B. et $\mathrm{N}$ arcy, $\mathrm{M}$., Présentation de la traduction de l'article de Lukasiewicz (1910b), 1991

Caujolle, F., «Logique et philosophie chez Jan Lukasiewicz », L'âge de la science, vol. III, $\mathrm{n}^{\circ} 1,1970$

Colin, P., L'audace et le soupçon, La crise du modernisme dans le catholicisme français (1893-1914), Paris, Desclée de Brouwer, 1997

$\mathrm{H}$ aack, S., D eviant L ogic, Fuzzy L ogic, Chicago, The University of Chicago Press, 1996

Lukasiewicz, J., O zasadnie sprzecznosci u Arystotelesa (1910a), Wolenski, J., dir., Varsovie, Panstwowie Wydawnictwo N aukowe, 1987 (traduction française inédite: Sikora, D., D u principe de contradiction chez A ristote).

- - «Über den Satz des Widerspruchs bei Arystoteles», Bulletin international de l'A cadémie des sciences de Cracovie, 1910b (traduction française: Cassin, B. et Narcy, M., "Sur le principe de contradiction chez Aristote», dans Rue Descartes, $n^{\circ} 1$ (Des Grecs), Paris, Albin M ichel, 1991)

- - , Jan Lukasiewicz, Selected Works, Borkowski, L., dir., Amsterdam, $\mathrm{N}$ orth-H olland, 1970

Pouivet, R., A près Wittgenstein, saint Thomas, Paris, Presses Universitaires de France, 1997

Puciato, R., « Thomism and M odern Formal Logic, Remarks on the Cracow Circle », Axiomathes, n.2, septembre 1993

Q uine, W.V., et Ullian, J.S., The Web of Belief, N ew York, Random H ouse, 1970

Skolimowski, H., Polish Analytical Philosophy, Londres, Routledge \& Kegan Paul, 1967

Smith, B., A ustrian Philosophy, The L egacy of Brentano, Chicago \& La Salle, O pen Court, 1994

Wolenski, J., Logic and Philosophy in the L ogic-Warsaw School, D ordrecht, Kluwer, 1989 